\title{
Molecular cytogenetic analysis and centromeric satellite organization of a novel $8 ; 11$ translocation in sheep: a possible intermediate in biarmed chromosome evolution
}

\author{
Raquel Chaves, ${ }^{1}$ Filomena Adega, ${ }^{1}$ Johannes Wienberg, ${ }^{2}$ Henrique Guedes-Pinto, ${ }^{1}$ John S. \\ Heslop-Harrison ${ }^{3}$ \\ ${ }^{1}$ Department of Genetics and Biotechnology, Centre of Genetics and Biotechnology/ICETA-UTAD, \\ University of Trá s-os-Montes and Alto Douro, P-5000-911Vila Real, Portugal \\ 2Institute of Human Genetics, GSF Forschungszentrum foer Umwelt und Gesundheit and Department \\ of Biology II, University of Munich, Richard-Wagner-Str., D-80333 Munich, Germany \\ ${ }^{3}$ Department of Biology, University of Leicester, Leicester LE1 7RH, UK
}

Correspondence to: R. Chaves; E-mail: rchaves@utad.pt

\begin{abstract}
During analysis of genome organization in sheep (Ovis aries, 2n = 54, XY/XX), we found a novel chromosomal translocation in an animal expected to be normal, adding to the six 'centric fusions' previously reported. The translocation was identified as $t(8 ; 11)$ by G-banding and was shown to be centric, involving whole chromosome arms by chromosome painting with probes for Chromosomes (Chrs) 8 and 11. Satellite I and a newly isolated satellite II clone was used to characterize the centromeric regions of both the novel and the three pairs of evolutionarily derived biarmed chromosomes. The novel $t(8 ; 11)$ showed satellite I proximal on both arms with satellite II covering the centromere, while the evolutionarily derived fusion leading to Chrs 2 and 3 showed the opposite configuration, not obviously derived by a simple fusion. Chr 1 has lost the satellite I hybridization patterns. The novel $\mathrm{t}(8 ; 11)$ pro- vides strong evidence for an intermediate step in evolution of the biarmed chromosomes in sheep.
\end{abstract}

Chromosomal fusions, usually regarded as Robertsonian, are a major feature of karyotype evolution in the Bovids, where the number of autosomal arms (NAA) 
remains constant at 60 . Sheep have $2 n=$ 54 with three pairs of biarmed chromosomes. A limited number of chromosomal rearrangements in Ovis aries have been reported in the literature. Centric fusion (Robertsonian) translocations are the most important and most extensively investigated chromosome abnormality of sheep. However, as far as we know only six "centric fusions" have been reported [according to the ISCNBD (2000) nomenclature, involving some renaming from the original publications; they are: t1(6;24) (Bruere 1969); t2(9;10), t3 $(7 ; 25)$ (Bruere et al. 1972); t4(5;8), t5(8;22) (Pearce et al. 1994); t2(6;24) or else $t(6 ; 26)$ (Murthy et al. 1995). There are three reports of reciprocal translocations [according to the ISCNBD (2000) no- menclature, they are: $\quad 1 p) ; 19 q+\quad$ Glahn-Luft and Wassmuth (1978), 13q);19q+ Anamthawat-Jonssó $\mathrm{n}$ et al. (1992), and 2q+;3q) Slota et al. (1986)].

Robertsonian translocations are thought to be the most common rearrangements in mammalian chromosomes. The study of the molecular etiology of Robertsonian translocations has concentrated on the analysis of the pericentric and short arm regions of the acrocentric chromosomes involved, since these are known to be the regions containing the translocation breakpoints.

Most mammalian chromosomes have satellite DNA (tandemly repeated DNA) located at or near the centromeres. Centromeric satellite probes have been isolated from many species of domesticated bovids (two-toed ungulates). Multiple satellites with different primary sequences have been identified in sheep, with the major fraction representing satellite I and II being used in the present study. These satel- lites are tandem arrays of sequences localized at the centromeres of all autosomes; however, there is less satellite I at the centromeres of biarmed autosomes, particularly at Chr 1 (Burkin et al. 1997; D'Aiuto et al. 1997; Chaves et al. 2000). Satellite II localizes on the acrocentric chromosomes and also at the $\mathrm{X} \mathrm{Chr} \mathrm{centromere.}$

We were initiating sheep lymphocyte cultures to obtain metaphases for analysis of genome organiza- tion. An unexpected karyotype, $2 \mathrm{n}=53, \mathrm{XY}$ was found, and here we report its characterization as a translocation. We analyzed the organization of the satellite I and II probes to enable modeling of the nature of chromosome fusions in biarmed chromosomes. The results may contribute to a better 
understanding of the formation of Robertsonian translocations. A possible pathway for the origin of the novel translocation and biarmed chromosomes is

\section{Materials and Methods}

\section{Chromosome preparations}

Peripheral blood samples were taken from two male sheep (wethers) for lymphocyte culture and chromosome preparation. No further sheep were sampled at this time: the samples were to be used as normal material for molecular cytogenetic analysis, and no morphological or behavioral abnormalities were noted in either sheep. They were part of a flock of the breed "Churra da Terra Quente" used by the University for teach- ing and research, managed following commercial practices, and they were sold before the results re- ported here were obtained. Chromosome preparations were made from short-term lymphocyte cultures of whole blood samples following standard methods (Chaves et al. 2002).

\section{CBP-banding.}

Air-dried slides were aged at $65 \mathrm{C}$ for 8 days and then submitted to C-banding following the standard barium hydroxide procedure of Sumner (1972), but using propidium iodide (PI) as stain presented, showing the dynamic nature of centromeric satellite organization and their importance in genome architecture and evolution.

(CBP-banding) (Fig. 1).

\section{GTG-banding and GTD-banding.}

Air-dried slides were aged at $65 \mathrm{C}$ for $6 \mathrm{~h}$ and then were submitted to standard Gbanding procedures (Verma and Babu 1995) with trypsin and staining with Giemsa (GTG-banding) (Fig. 2).

For the fluorescent in situ hybridization (FISH) experiments, all the chromosome preparations were submitted to prior G-banding procedures with trypsin, but the slides were not stained at this step. In- stead, the slides were fixed in paraformaldehyde as described by Chaves et al. (2002) and stained with 4', 6'-diamidino-2phenylindole (DAPI) (GTD-banding) (Figs.3, 4). Chromosome identification and karyotypes were analyzed according to the recommendations for the standardization of the Domestic Bovids karyotypes (ISCNDB 2000).

DNA probes and Fluorescent In Situ Hybridization (FISH). Chromosome painting of sheep chromosomes was performed in a first round of FISH experiments with two sheep painting 
probes representing $\mathrm{Chr} 8$ and 11 from Ovis aries (Burkin et al. 1997). Briefly, $15 \mathrm{LL}$ of the hybridization mixture (50\% formamide, 2- SSC, 10\% dextran sulfate, with $500 \mathrm{ng}$ of each paint probe, $5 \mathrm{lg}$ of sonicated sheep genomic DNA, $10 \mathrm{lg}$ of salmon sperm DNA) was denatured at $70 \mathrm{C}$ for $10 \mathrm{~min}$, allowed to preanneal for $90 \mathrm{~min}$ at $37 \mathrm{C}$, dropped on denatured chromosome preparations, and mounted with $22 \times 22 \mathrm{~mm}$ cover slips. Slides were denatured in $70 \%$ formamide, 2- SSC at $65 \mathrm{C}$ for $30 \mathrm{~s}$. In situ hybridization was performed for $48 \mathrm{~h}$ at 37 C. Detection of the hybridization signal was as published (Chaves et al. 2002) with the most stringent wash being in $50 \%$ formamide, $2 \times \mathrm{SSC}$ at $42 \mathrm{C}$. After hybridization and washing of the slides, digoxigeninlabeled chromosome paints were detected with 5-carboxy-tetramethylrhodamine (5-TAMRA) conjugated to anti-digoxigenin (Roche), and biotin-labeled probes were detected with avidin conjugated to fluorescein isothiocyanate (FITC) (Vector Laboratories, Vector). Chromosomes were counterstained with DAPI and mounted in Vectashield (Vector Laboratories, Vector). After image acquisition, the slides were washed in $4 \times$ SSC, $0.05 \%$ Tween 20 at room temperature for $5 \mathrm{~h}$ with agitation, dehydrated, and denatured as described above, following a second round of FISH experiment with satellite I and II sheep clones.

A satellite I sheep clone (pOaKB9; Chaves et al. 2000) and a satellite II sheep clone described here (named cOaGBII-1) were used as probes. They were labeled with either biotin-16-dUTP (Sigma) or digoxigenin-11-dUTP by standard PCR procedures (Schwarzacher and Heslop-Harrison 2000). The cOaGBII-1 sheep clone was prepared from sheep genomic DNA PCR amplification with specific primers $\left(5^{\prime}\right)$ GTTGCACATCCAAGGGCTCC-3' and 5'-CCGGCAGAGCAGCCTCGC-3'

designed from species-homologous sequences in GenBank. Briefly, $50 \mathrm{ng}$ template DNA was amplified with the primers in $501 \mathrm{~L}$ reaction buffer with 1.5 $\mathrm{mM} \mathrm{MgCl}_{2}, 0.2 \mathrm{mM}$ dNTPs, and 1.25 U Taq polymerase (Fermentas) by using predenaturation for $2 \mathrm{~min}$ at $93 \mathrm{C}$, then 30 cycles of $45 \mathrm{~s}$ at $92 \mathrm{C}, 45 \mathrm{~s}$ at 60 C, and $45 \mathrm{~s}$ at $72 \mathrm{C}$. A fragment of 1200 bp was purified and cloned in the pCR 4TOPO vector (Invitrogen). cOaGBII-1 was partially sequenced and showed more than $95 \%$ homology with previously isolated sheep satellite II sequences (e.g., EMBO mammalian database accessions U24094, U24093, U24092). 
The FISH experiments with the DNA satellite I and II probes were performed with $151 \mathrm{~L}$ of the hybridization mixture (50\% formamide, $2 \times \mathrm{SSC}, 10 \%$ dextran sulfate, $100 \mathrm{ng}$ of each DNA satellite probe) per slide. Subsequent FISH procedures were as above.

\section{Chromosome observation}

Chromosomes were analyzed with a Zeiss Axioplan 2 Imaging with an Axiocam digital camera and AxioVision software. Digitized photos were prepared for printing in Adobe Photoshop (version 5.0); contrast, overlaying, and color optimization were the functions used, and all affected the whole of the image equally

metaphases of the sheep. Satellite I hybridization was undetectable in the centromeric region (Fig. $3 b, f, d, h)$ of biarmed Chr 1 (along with the $\mathrm{X} \mathrm{Chr),}$ while biarmed $\mathrm{Chr} 2$ and 3 showed satellite I (Fig. 3b, f, d, h) hybridization at a single centromeric site flanked by larger arrays of the satellite II (Fig. 3c, g, d, h) sequence. The monobrachial autosomal chromosomes show distal sites of satellite I with proximal arrays of satellite II (as reported by D'Aiuto et al. 1997). In contrast to the other biarmed chromosomes, the novel $\mathrm{t}(8 ; 11)$ chromosome showed a single array of the satellite II (Fig. 3c, g, d, h) sequence at the centromere, flanked by arrays of the satellite I (Fig. 3b, f, d, h) sequence. The different localization of satellites I and II in the novel $\mathrm{t}(8 ; 11)$ chromosome and in the biarmed $\mathrm{Chr} 2,3$, and 1 is particularly clear (Fig. 4). D'Aiuto et 
al. (1997) found hybridization patterns for their satellite II probes slightly different from the biarmed chromosomes, with localization on only one side of the centromere, probably a consequence of small differences in the probe sequence and hybridization stringency.

Translocation cases in sheep are apparently rare, since only six different fusion chromosomes have been reported, and they occur in single breeds (or where there is evidence of cross-breeding) (Pearce et al. 1994). Given the low economic value of indi- vidual sheep and the high cull rate at young ages, it is unlikely that cytogenetic investigations are carried out in many cases of genetic abnormalities or fertility problems, although if translocations were very frequent, one might expect such abnormalities would be reported from University flocks.

Contrasting with the three other biarmed autosomes, the novel $\mathrm{t}(8 ; 11)$ showed a remarkable organization of its centromeric satellite sequences. Both the $\mathrm{C}$ and G-banding investigations would have suggested each biarmed chromosome arose from a simple fusion of two acrocentric chromosomes with distal satellite II and proximal satellite I. However, the satellite hybridization pattern shows that the evolution after fusion was more complex. The blocks of centromeric satellites are rearranged in order in Chr 1, 2, and 3 but maintain a fused pattern in $t(8 ; 11)$. Thus, the newly identified $\mathrm{t}(8 ; 11)$ chromosome shows a different organization from the biarmed chromosomes that are evolutionarily derived. If the ancestral satellite patterns of all acrocentric autosomes were similar, then the biarmed Chr 1, 2, and 3 have undergone a complex reorganization of the satellite I and II sequences during chromosomal fusion and evolution. Whether the novel $\mathrm{t}(8 ; 11)$ is de novo or a stable polymorphism in this sheep breed is not known, but, regardless, it provides strong evidence for an evolutionary inter-mediate step (Fig. 5). Examination of other sheep breeds and species in the genus Ovis or tribe Caprini with different numbers of biarmed autosomes will provide insight into frequencies, organization, and evidence for the mechanisms of evolution of fused chromosomes in this group.

\section{Acknowledgments}

This work was supported by two grants-PRAXIS XXI/BD/9046/96 and SFRH/BD/3280/2000 — and a project, Molecular Cytogenetic Evolution in the 
POCTI/P/BIA/11285/98. We thank M.A.

Ferguson-Smith and D. Burkin for the chromosome sheep paint probes. J.S. Heslop-Harrison thanks CREST, Japan Science and Technology Agency, for support of centromeric sequence work.

\section{References}

1. Anamthawat-Jonssón K, Long SE, Basrur PK, Adal- steinsson S (1992) Reciprocal translocation $(13 ; 20)(\mathrm{q} 12 ; \mathrm{q} 22)$ in an Icelandic sheep. Res Vet Rec 52, 367-370

2. Bruere AN (1969) Male sterility and an autosomal translocation in Romney sheep. Cytogenetics 8, 209-218

3. Bruere AN, Chapman HM, Wyllie DR (1972) Chro- mosome polymorphism and its implications in the select Drysdale breed of sheep. Cytogenetics 11, 233-246.

4. Burkin DJ, O'Brien PCM, Broad TE, Hill DF, Jones CA et al. (1997) Isolation of chromosome-specific paints from highresolution flow karyotypes of the sheep (Ovis aries). Chromosome Res 5, 102-108.

5. Chaves R, Guedes-Pinto H, Heslop-Harrison JS, Schwarzacher T (2000) The species and chromosomal distribution of the centromeric a-satellite I sequence from sheep in the tribe Caprini and other Bovidae. Cytogenet Cell Genet 91, 62-66

6. Chaves R, Adega F, Santos S, HeslopHarrison JS, Guedes-Pinto H (2002) In situ hybridization and chromosome banding in mammalian species. Cytogenet Genome Res 96, 113-116
7. D'Aiuto L, Barsanti P, Mauro S, Cserpan I, Lavane C, Ciccarese S (1997) Physical relationship between satellite I and II DNA in centromeric regions of sheep chromosomes. Chromosome Res 5, 375381

8. Glahn-Luft B, Wassmuth R (1978) Gbanding in sheep with heterozygous and homozygous translocations. Ann Ge né t Sél Anim 10, 594

9. ISCNDB (2000) International system for chromosome nomenclature of domestic bovids. Di Berardino D, Di Meo GP, Gallagher DS, Hayes H, Iannuzzi L (coordi- nator) (eds). Cytogenet Cell Genet 92, 283-299 (2001)

10. Murthy SRS, Jayashankar MR, Nagaraja CS, Govinda- iah MG, Sridhara-Murthy SR (1995) Association of cryptorchidism with autosomal translocation in Deccani sheep. Ind J Small Rum 1, 47-48

11. Pearce PD, Ansari HA, Maher DW, Malcolm AA, Ste- wart-Scott IA et al. (1994) New Robertsonian translo- cation chromosomes in domestic sheep (Ovis aries). Cytogenet Cell Genet 67, 137-140

12. Schwarzacher T, Heslop-Harrison P (2000) Practical in situ Hybridization. (Oxford: Bios) 
13. Slota F, Danielak B, Kozubska A (1986) Structural rearrangement of metacentric chromosomes in the ram of the Polish Wrzosowka breed. In Abstracts of the 7 th Eur Coll Cytogenet Domestic Animals, Warsaw, Poland, 37

14. Sumner AT (1972) A simple technique for demon- strating centromeric heterochromatin. Exp Cell Res

75, 304-306

15. Verma RS, Babu (1995) Human Chromosomes:

Principles and Techniques. (New York: McGraw Hill. 


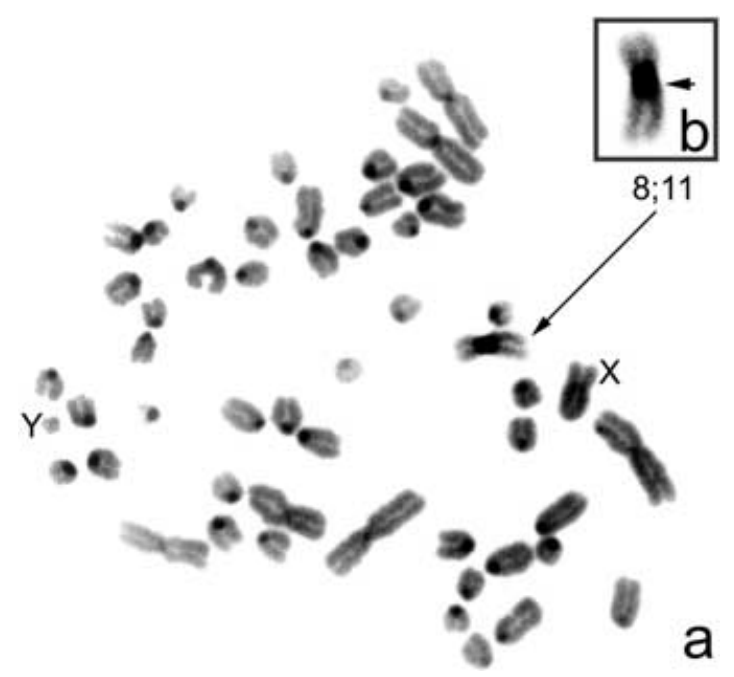

Fig. 1. (a) C-banded metaphase of a sheep with $2 n=53$, XY. A single fusion chromosome-8;11-is seen with a large block of heterochromatin (enlarged insert, $b$ ).
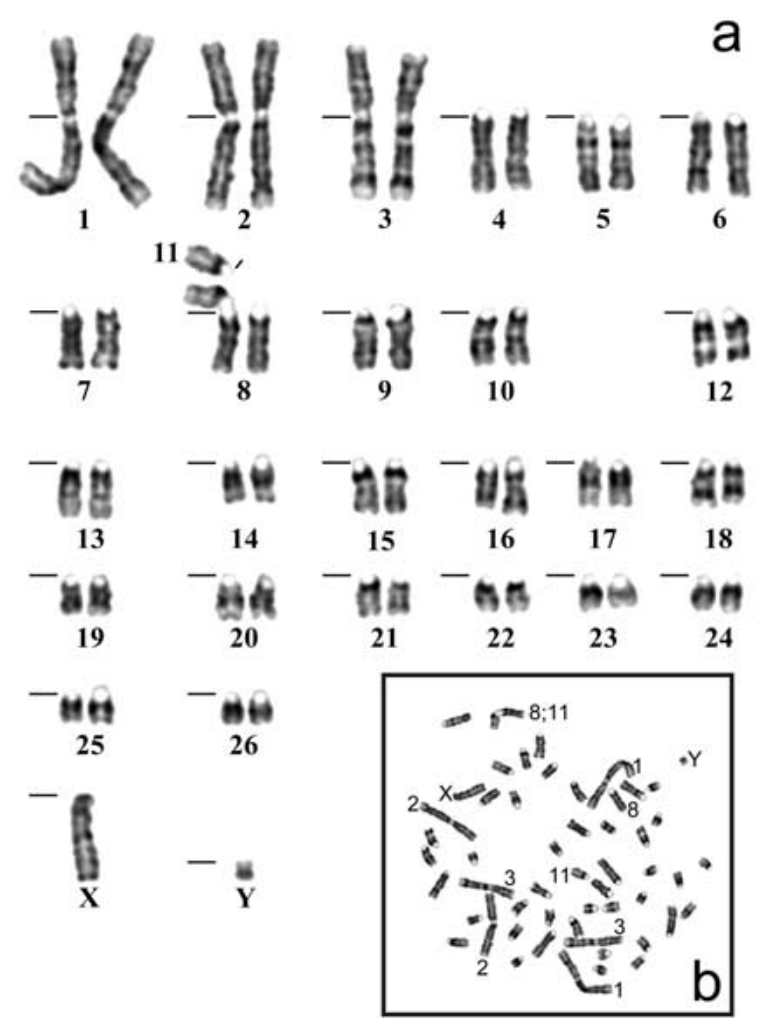

Fig. 2. (a) Karyotype of a sheep with $2 \mathrm{n}=53, \mathrm{XY}$ and (b) G- banded metaphase showing the $\mathrm{Chr} 1,2,3,8,11,8$; 11 fusion, and the sex chromosomes 

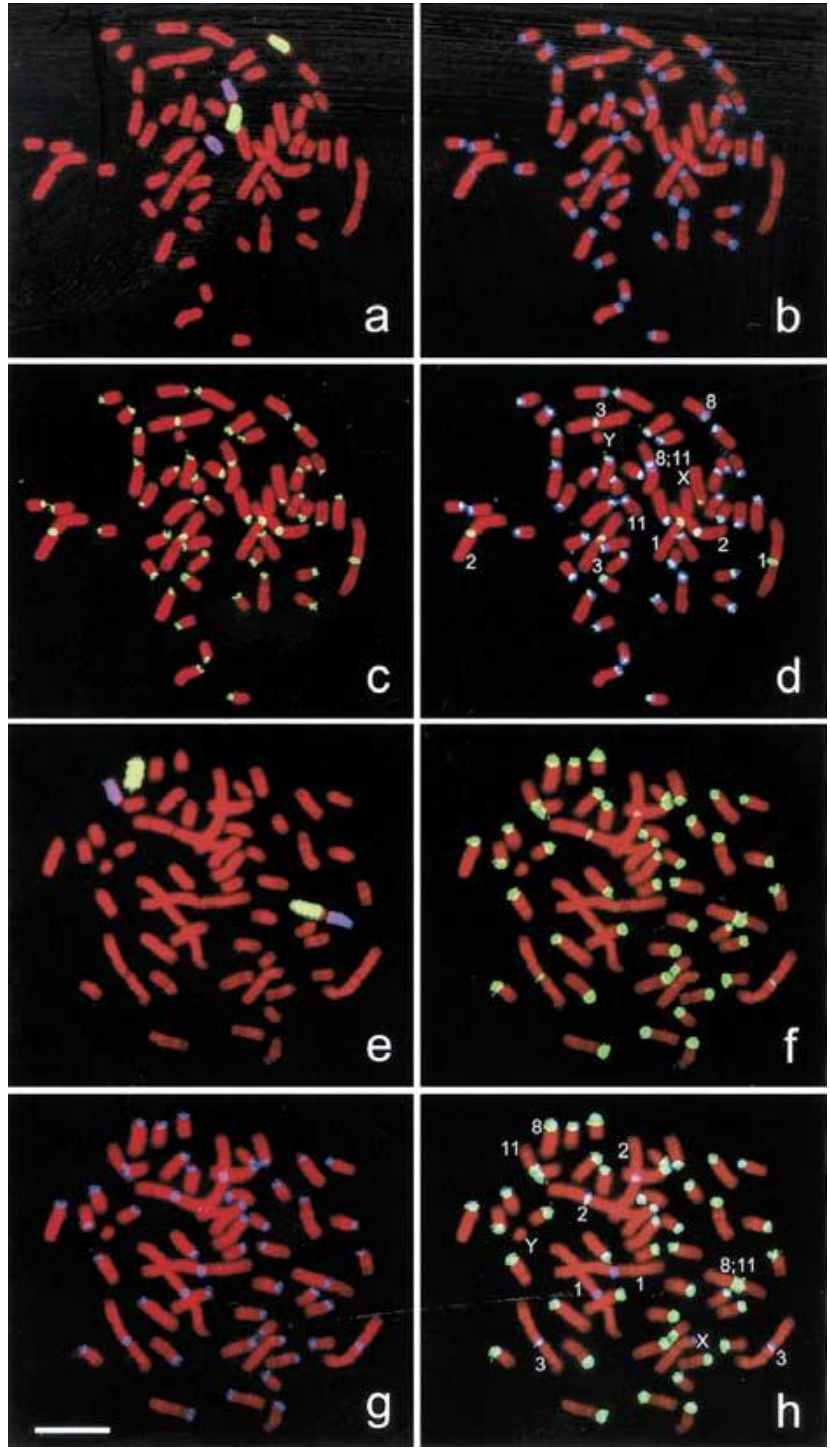

Fig. 3. Two $2 \mathrm{n}=53$, $\mathrm{XY}$ metaphases $(\mathrm{a}-\mathrm{d}$ and $\mathrm{e}-\mathrm{h}$ with chromosomes red in false color DAPI staining) showing chromosome paints for 8 (yellow) and 11 (magenta; parts a, e), and in situ hybridization of satellite I (cyan b, and yel- low f), satellite II (yellow c, and cyan g), and superimposed with identification of key chromosomes (d, h). Scale bar in g represents $101 \mathrm{~m}$. 


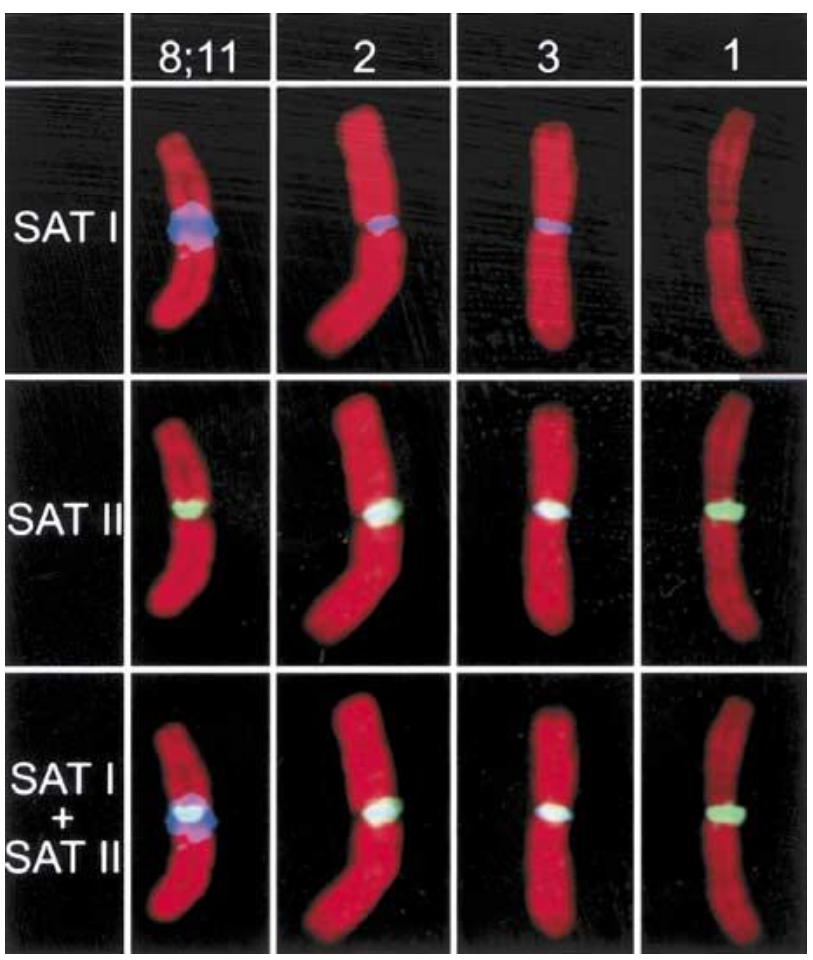

Fig. 4. Image showing a detailed comparison of the in situ hybridization with satellite I and II probes in the biarmed chromosomes of the sheep with $2 \mathrm{n}=53$, XY. The biarmed Chr 2, 3, and 1 are ordered from the most recent to the postulated evolutionarily oldest chromosome, following the model in Fig. 5 where the biarmed chromosomes arise sequentially by fusion of monobrachial chromosomes.

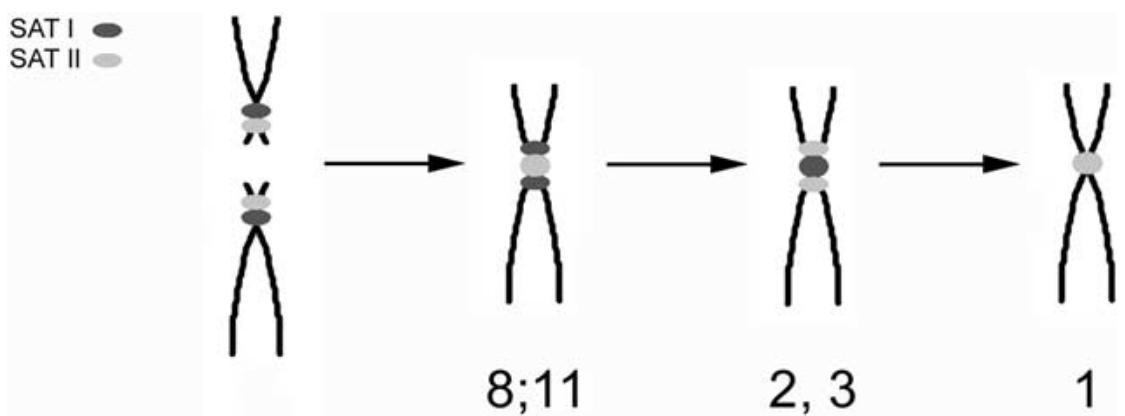

Fig. 5. An evolutionary model showing the $8 ; 11$ chromosome as an intermediate in the sheep biarmed chromosome evolution 